\title{
APLICACIÓN WEB PARA ESTIMACIÓN DE ATENUACIÓN POR LLUVIA EN ENLACES CON EL SATÉLITE TKSAT-1
}

Gustavo Siles, Gustavo Marín

\section{RESUMEN}

En el presente artículo se introduce una aplicación web diseñada para el cálculo de estadísticas de atenuación por lluvia en Bolivia. Los cálculos que realiza son válidos para enlaces con el satélite boliviano TKSat-1 y se realizan en base la últimas Recomendaciones proporcionadas por la UIT-R. Se presentan un par de aplicaciones concretas tomando en cuenta dos emplazamientos en Bolivia: uno en una región tropical con altos niveles de precipitación, y otro en una región altiplánica en la región occidental del país. Los resultados obtenidos demuestran la utilidad de la aplicación y permiten estudiar de manera cuantitativa los diferentes efectos que produce la lluvia a diferentes bandas de frecuencias, así como su comportamiento estadístico en un año medio.

Palabras Clave: Comunicaciones Satelitales, Atenuación por Lluvia, Propagación, Satélite TKSat-1.

DOI: 10.23881 idupbo.020.1-7i 